\title{
Public health support to policies in the fields of headache. Different ways of producing data and modalities of reading them with the aid of the meta-analytic approach
}

Received: 5 May 2006

Accepted in revised form: 27 May 2006

Published online: 15 June 2006
Decision-makers need the best available evidence in matters of interest to them. Obtaining it means systematically identifying and assembling all the relevant evidence then available, and assessing its quality, limitations and uncertainty in order to use it to the best effect in producing quantity estimates. "Best available", however, is not always good. Nonetheless, concerns about poor quality or uncertainty of data are not an acceptable reason for rejecting such evidence as decisions must often be made now: decision-makers cannot and will not wait years for improvements in evidence.

Policy-makers, their partner public-health experts and consumers alike seek rational guides to set priorities for health care in a context of limited resources. They need to evaluate the outcomes of a range of interventions and health-care reforms, monitoring changes over time at local, national, regional or global levels. Clearly, estimation of needs for health services and of the costs and effectiveness of these services requires indicators that go beyond measures of death rates or diagnosis alone and take into account the effects of disease and health care upon people's ability to function normally.

As Dr Buzzi, the author of the paper in this issue of the journal on public health, says "... information is often sought by reading reviews that summarise data from original articles. However, such a time-saving learning method, may, somehow, drive the attention towards certain parameters and obscure other features, according to the purpose of a given Author and therefore provide partial information on a given issue. To obviate the risk of partial or driven information, the meta-analytic method has been proposed in order to revise and analyse data with statistical aids from large numbers of original trials and to provide objective conclusions."

As Bero and colleagues say [1, 2], systematic reviews and metaanalyses are two means for looking at a range of studies using specific criteria and methods and consumers and policymakers can use systematic reviews for decision making. "A systematic review is an overview of primary studies which contains an explicit statement of objectives, materials, and methods and has been conducted according to explicit and reproducible methodology" [3].

According to McKibbon [4], systematic reviews are conducted to answer a narrow and more focused question about effectiveness [9]. Some advantages of systematic reviews, presented by Greenhalgh [3], are presented in Table 1.

To conduct a systematic review requires extensive time and resource 
Table 1 Advantages of systematic reviews [3]

Explicit methods limit bias in identifying and rejecting studies.

Conclusions are more reliable and accurate because of methods used.

Large amounts of information can be assimilated quickly by health care providers, researchers and policy makers.

Delay between research discoveries and implementation of effective diagnostic and therapeutic strategies may be reduced.

Results of different studies can be formally compared to establish generalisability of findings and consistency (lack of heterogeneity) of results.

Reasons for heterogeneity (inconsistency in results across studies) can be identified and new hypotheses generated about particular subgroups.

Quantitative systematic reviews (meta-analyses) increase the precision of the overall result.

Table 2 Steps for conducting a systematic review [4]

\begin{tabular}{ll}
\hline Step & Comment \\
\hline Step 1: Problem formulation & $\begin{array}{l}\text { This includes not only the question to be answered from the review but also the } \\
\text { interventions, populations, settings, outcomes, duration, and the inclusion and exclusion } \\
\text { criteria to be considered in selecting individual studies for review. } \\
\text { This step is becoming more rigorous. Multiple techniques or processes } \\
\text { are necessary to identify relevant literature. A comprehensive, thorough and carefully } \\
\text { planned search strategy is required to locate published as well as grey literature. } \\
\text { Step 2: Identifying and selecting studies } \\
\text { Computer and hand searching will identify studies to be examined according to inclusion } \\
\text { and exclusion criteria. }\end{array}$ \\
$\begin{array}{l}\text { Using a tabular format for extracting the data from a study helps the reviewer understand } \\
\text { the range of studies, assess the data and report results. This process is often completed } \\
\text { in duplicate or triplicate to compare results for consistency. }\end{array}$ \\
$\begin{array}{l}\text { Researchers determine whether the data are similar enough to be combined } \\
\text { mathematically and statistically. If the data can be combined, meta-analysis may } \\
\text { be possible. } \\
\text { Data and results are generally presented in several formats. Raw data from each study } \\
\text { Step 5: Presentation of the results }\end{array}$ \\
$\begin{array}{l}\text { are usually presented in table form. These data are accompanied by written comparisons } \\
\text { among the studies. }\end{array}$ \\
\hline
\end{tabular}

commitment. McKibbon [4] outlines five specific steps for producing a systematic review article. These steps are summarised in Table 2.

Greenhalgh [3] concurs that when a systematic review is conducted, there are two important components. First, the search for relevant articles must be thorough and objective.

Second, and equally as important, the criteria used to exclude articles must be explicit and independent of the results of the research. This is clearly highlighted in the approach of Dr Buzzi's paper and it is "migraine and triptans related".

The most enduring and useful systematic reviews, notably those undertaken by the Cochrane Collaboration, are regularly updated to incorporate new evidence [5].

Dr Buzzi's paper should not be considered as a review, as it does not take into consideration the whole literature concerning triptans, the author, as she says of herself, "not being prone to such a titanic enterprise". Rather, it makes some examples of different ways of producing data and tries to discuss the modalities of reading them to avoid misinterpretation and misuse of collected information.

Over the past 15 years important epidemiological papers have produced a culture of the public health importance of headaches and their clinical, economic and humanistic 
impact on people and society, joining all the work done at basic research and pathophysiological levels [6-8].

The contemporary appearance at international level of the concept of "sustainable medicine", in terms of allocation of the available health resources, unfortunately not matched to the high levels of headache-related health care needs, is leading researchers in the headache field to involve and work closely with health economists, public health administrators and lay organisations to co-operate in developing ideas for best practice to meet these needs.

Finding a common methodology to support this process is one of the aims of Dr Buzzi's paper in the specific field of migraine and triptans, and might be considered a support for the increasing awareness process that is a necessary step in the field of headache.

Section Editor: M. Leonardi HEADNET Research Group: Public Health, Disability, ICF EU-MHADIE Project Coordinator Scientific Coordinator and WHO Liaison "ICF in Italy Project" Scientific Direction Italian National Neurological Institute C. Besta,

Via Celoria 11, I-20133 Milan, Italy e-mail:leonardi@istituto-besta.it web: www.mhadie.it

\section{References}

1. Bero LA, Jadad AR (1997) How consumers and policymakers can use systematic reviews for decision making. Ann Intern Med 127:37-42

2. Bero LA, Grilli R, Grimshaw JM, Harvey E, Oxman AD, Thomson MA (1998) Closing the gap between research and practice: an overview of systematic reviews of interventions to promote the implementation of research findings. Br Med J 317:465-468
3. Greenhalgh T (1997) How to read a paper: papers that summarise other papers (systematic reviews and metaanalyses). Br Med J 315:672-675

4. McKibbon A (1999) PDQ evidencebased principles and practice. B.C. Decker, Inc., Saint Louis

5. Cochrane Collaboration (2001) The ten principles of the Cochrane collaboration [online]. Available at http://www.cochrane.org/docs/ tenprinciples.htm

6. Martelletti P, Leonardi M (2003) The global impact of migraine. J Headache Pain 4[Suppl 1]:S1-S3
7. Leonardi M (2003) Migraine and disability: WHO's work to measure functioning, disability and health and the Global Burden of Diseases Study. J Headache Pain 4[Suppl 1]:S12-S18

8. Leonardi M, Steiner TJ, Scher AI, Lipton RB (2005) The Global Burden of migraine: measuring disability in headache disorders with WHO's Classification and Functioning, Disability and Health (ICF). J Headache Pain 6:429-440

9. Hunt DL, McKibbon KA (1997) Locating and appraising systematic reviews. Ann Intern Med 126:532-538 